\title{
Singularly Perturbed Monotone Systems and an Application to Double Phosphorylation Cycles
}

\author{
Liming Wang and Eduardo Sontag*†
}

July 10, 2018

\begin{abstract}
The theory of monotone dynamical systems has been found very useful in the modeling of some gene, protein, and signaling networks. In monotone systems, every net feedback loop is positive. On the other hand, negative feedback loops are important features of many systems, since they are required for adaptation and precision. This paper shows that, provided that these negative loops act at a comparatively fast time scale, the main dynamical property of (strongly) monotone systems, convergence to steady states, is still valid. An application is worked out to a double-phosphorylation "futile cycle" motif which plays a central role in eukaryotic cell signaling.
\end{abstract}

\section{Introduction}

Monotone dynamical systems constitute a rich class of models, for which global and almost-global convergence properties can be established. They are particularly useful in biochemical applications and also appear in areas like coordination [28] and other problems in control [7]. One of the fundamental results in monotone systems theory is Hirsch's Generic Convergence Theorem [17, 18, 19, 37]. Informally stated, Hirsch's result says that almost every bounded solution of a strongly monotone system converges to the set of equilibria. There is a rich literature regarding the application of this powerful theorem, as well as of other results dealing with everywhere convergence when equilibria are unique ([9, 22, 37]), to models of biochemical systems. See for instance [39, 40] for expositions and many references.

Unfortunately, many models in biology are not monotone, at least with respect to any standard orthant order. This is because in monotone systems (with respect to orthant orders) every net feedback loop should be positive, but, on the other hand, in many systems negative feedback loops often appear as well, as they are required for adaptation and precision. However, intuitively, negative loops that act at a comparatively fast time scale should not affect the main characteristics of monotone behavior. The main purpose of this paper is to show that this is indeed the case, in the sense that singularly perturbed strongly monotone systems inherit generic convergence properties. A system that is not monotone may become monotone once that fast variables are replaced by their steady-state values. In order to prove a precise time-separation result, we employ tools from geometric singular perturbation theory.

This point of view is of special interest in the context of biochemical systems; for example, Michaelis Menten kinetics are mathematically justified as singularly perturbed versions of mass action kinetics [11, 29. One particular example of great interest in view of current systems biology research is that of "futile cycle" motifs, as illustrated in Figure 1. As discussed in [34, futile cycles (with any number of intermediate

\footnotetext{
${ }^{*}$ L. Wang is with the Rutgers University, Department of Mathematics, wshwlm@math.rutgers.edu.

${ }^{\dagger}$ E. Sontag is with the Rutgers University, Department of Mathematics, sontag@math.rutgers.edu.
} 


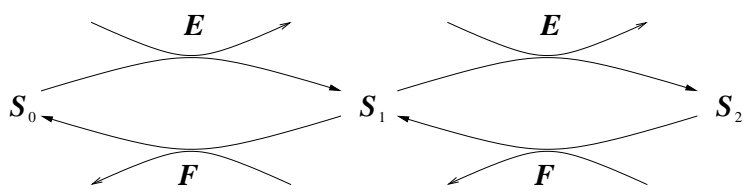

Figure 1: Dual futile cycle. A substrate $S_{0}$ is ultimately converted into a product $P$, in an "activation" reaction triggered or facilitated by an enzyme $E$, and, conversely, $P$ is transformed back (or "deactivated") into the original $S_{0}$, helped on by the action of a second enzyme $F$.

steps, and also called substrate cycles, enzymatic cycles, or enzymatic interconversions) underlie signaling processes such as GTPase cycles [10], bacterial two-component systems and phosphorelays [3, 15] actin treadmilling [6]), and glucose mobilization [24, as well as metabolic control [41] and cell division and apoptosis [42] and cell-cycle checkpoint control [26]. A most important instance is that of Mitogen-Activated Protein Kinase ("MAPK") cascades, which regulate primary cellular activities such as proliferation, differentiation, and apoptosis [2, 5, 20, 46] in eukaryotes from yeast to humans. MAPK cascades usually consist of three tiers of similar structures with multiple feedbacks [4, [13, 47]. Here we focus on one individual level of a MAPK cascade, which is a futile cycle as depicted in Figure 1. The precise mathematical model is described later. Numerical simulations of this model have suggested that the system may be monostable or bistable, see [27]. The later will give rise to switch-like behavior, which is ubiquitous in cellular pathways ([14, 32, 35, 36]). In either case, the system under meaningful biological parameters shows convergence, not other dynamical properties such as periodic behavior or even chaotic behavior. Analytical studies done for the quasi-steady-state version of the model (slow dynamics), which is a monotone system, indicate that the reduced system is indeed monostable or bistable, see [31]. Thus, it is of great interest to show that, at least in certain parameter ranges (as required by singular perturbation theory), the full system inherits convergence properties from the reduced system, and this is what we do as an application of our results.

A feature of our approach, as in other control problems [1, 21], is the use of geometric invariant manifold theory [12, 23, 30]. There is a manifold $M_{\varepsilon}$, invariant for the full dynamics of a singularly perturbed system, which attracts all near-enough solutions. However, we need to exploit the full power of the theory, and especially the fibration structure and an asymptotic phase property. The system restricted to the invariant manifold $M_{\varepsilon}$ is a regular perturbation of the slow $(\varepsilon=0)$ system. As remarked in Theorem 1.2 in Hirsch's early paper [17, a $C^{1}$ regular perturbation of a flow with eventually positive derivatives also has generic convergence properties. So, solutions in the manifold will generally be well-behaved, and asymptotic phase implies that solutions near $M_{\varepsilon}$ track solutions in $M_{\varepsilon}$, and hence also converge to equilibria if solutions on $M_{\varepsilon}$ do. A key technical detail is to establish that the tracking solutions also start from the "good" set of initial conditions, for generic solutions of the large system.

A preliminary version of these results was presented at the 2006 Conference on Decision and Control, and dealt with the special case of singularly perturbed systems of the form:

$$
\begin{aligned}
\dot{x} & =f(x, y) \\
\varepsilon \dot{y} & =A y+h(x)
\end{aligned}
$$

on a product domain, where $A$ is a constant Hurwitz matrix and the reduced system $\dot{x}=f\left(x,-A^{-1} h(x)\right)$ is strongly monotone. However, for the application to the above futile cycle, there are two major problems with that formulation: first, the dynamics of the fast system have to be allowed to be nonlinear in $y$, and second, it is crucial to allow for an $\varepsilon$-dependence on the right-hand side as well as to allow the domain to be a polytope depending on $\varepsilon$. We provide a much more general formulation here.

We note that no assumptions are imposed regarding global convergence of the reduced system, which 
is essential because of the intended application to multi-stable systems. This seems to rule out the applicability of Lyapunov-theoretic and ISS tools [8, 43].

This paper is organized as follows. The main result is stated in Section 2. In Section 3, we review some basic definitions and theorems about monotone systems. The detailed proof of the main theorem can be found in Section 4, and applications to the MAPK system and another set of ordinary differential equations are discussed in Section 5. Finally, in Section 6, we summarize the key points of this paper.

\section{Statement of the Main Theorem}

In this paper, we focus on the dynamics of the following prototypical system in singularly perturbed form:

$$
\begin{aligned}
\frac{d x}{d t} & =f_{0}(x, y, \varepsilon) \\
\varepsilon \frac{d y}{d t} & =g_{0}(x, y, \varepsilon) .
\end{aligned}
$$

We will be interested in the dynamics of this system on a time-varying domain $D_{\varepsilon}$. For $0<\varepsilon \ll 1$, the variable $x$ changes much slower than $y$. As long as $\varepsilon \neq 0$, one may also change the time scale to $\tau=t / \varepsilon$, and study the equivalent form:

$$
\begin{aligned}
& \frac{d x}{d \tau}=\varepsilon f_{0}(x, y, \varepsilon) \\
& \frac{d y}{d \tau}=g_{0}(x, y, \varepsilon) .
\end{aligned}
$$

Within this general framework, we will make the following assumptions (some technical terms will be defined later), where the integer $r>1$ and the positive number $\varepsilon_{0}$ are fixed from now on:

A1 Let $U \subset \mathbb{R}^{n}$ and $V \subset \mathbb{R}^{m}$ be open and bounded. The functions

$$
\begin{gathered}
f_{0}: U \times V \times\left[0, \varepsilon_{0}\right] \rightarrow \mathbb{R}^{n} \\
g_{0}: U \times V \times\left[0, \varepsilon_{0}\right] \rightarrow \mathbb{R}^{m}
\end{gathered}
$$

are both of class $C_{b}^{r}$, where a function $f$ is in $C_{b}^{r}$ if it is in $C^{r}$ and its derivatives up to order $r$ as well as $f$ itself are bounded.

A2 There is a function

$$
m_{0}: U \rightarrow V
$$

of class $C_{b}^{r}$, such that $g_{0}\left(x, m_{0}(x), 0\right)=0$ for all $x$ in $U$.

It is often helpful to consider $z=y-m_{0}(x)$, and the fast system (2) in the new coordinates becomes:

$$
\begin{aligned}
& \frac{d x}{d \tau}=\varepsilon f_{1}(x, z, \varepsilon) \\
& \frac{d z}{d \tau}=g_{1}(x, z, \varepsilon)
\end{aligned}
$$


where

$$
\begin{aligned}
& f_{1}(x, z, \varepsilon)=f_{0}\left(x, z+m_{0}(x), \varepsilon\right), \\
& g_{1}(x, z, \varepsilon)=g_{0}\left(x, z+m_{0}(x), \varepsilon\right)-\varepsilon\left[D_{x} m_{0}(x)\right] f_{1}(x, z, \varepsilon) .
\end{aligned}
$$

When $\varepsilon=0$, the system (3) degenerates to

$$
\frac{d z}{d \tau}=g_{1}(x, z, 0), \quad x(\tau) \equiv x_{0} \in U
$$

seen as equations on $\left\{z \mid z+m_{0}\left(x_{0}\right) \in V\right\}$.

A3 The steady state $z=0$ of (4) is globally asymptotically stable on $\left\{z \mid z+m_{0}\left(x_{0}\right) \in V\right\}$ for all $x_{0} \in U$.

A4 All eigenvalues of the matrix $D_{y} g_{0}\left(x, m_{0}(x), 0\right)$ have negative real parts for every $x \in U$, i.e. the matrix $D_{y} g_{0}\left(x, m_{0}(x), 0\right)$ is Hurwitz on $U$.

A5 There exists a family of convex compact sets $D_{\varepsilon} \subset U \times V$, which depend continuously on $\varepsilon \in\left[0, \varepsilon_{0}\right]$, such that (11) is positively invariant on $D_{\varepsilon}$ for $\varepsilon \in\left(0, \varepsilon_{0}\right]$.

A6 The flow $\psi_{t}^{0}$ of the limiting system (set $\varepsilon=0$ in (11)):

$$
\frac{d x}{d t}=f_{0}\left(x, m_{0}(x), 0\right)
$$

has eventually positive derivatives on $K_{0}$, where $K_{0}$ is the projection of

$$
D_{0} \bigcap\left\{(x, y) \mid y=m_{0}(x), x \in U\right\}
$$

onto the $x$-axis.

A7 The set of equilibria of (1) on $D_{\varepsilon}$ is totally disconnected.

Remark 1 Assumption A3 implies that $y=m_{0}(x)$ is a unique solution of $g_{0}(x, y, 0)=0$ on $U$.

Continuity in $\mathbf{A 5}$ is understood with respect to the Hausdorff metric.

In mass-action chemical kinetics, the vector fields are polynomials. So, A1 follows naturally.

Our main theorem is:

Theorem 1 Under assumptions $\mathbf{A} 1$ to $\mathbf{A} 7$, there exists a positive constant $\varepsilon^{*}<\varepsilon_{0}$ such that for each $\varepsilon \in$ $\left(0, \varepsilon^{*}\right)$, the forward trajectory of (1) starting from almost every point in $D_{\varepsilon}$ converges to some equilibrium.

\section{Monotone Systems of Ordinary Differential Equations}

In this section, we review several useful definitions and theorems regarding monotone systems. As we wish to provide results valid for arbitrary orders, not merely orthants, and some of these results, though well-known, are not readily available in a form needed for reference, we provide some technical proofs.

Definition 1 A nonempty, closed set $C \subset \mathbb{R}^{N}$ is a cone if 
1. $C+C \subset C$,

2. $\mathbb{R}_{+} C \subset C$,

3. $C \bigcap(-C)=\{0\}$.

We always assume $C \neq\{0\}$. Associated to a cone $C$ is a partial order on $\mathbb{R}^{N}$. For any $x, y \in \mathbb{R}^{N}$, we define:

$$
\begin{aligned}
& x \geq y \Leftrightarrow x-y \in C \\
& x>y \Leftrightarrow x-y \in C, x \neq y .
\end{aligned}
$$

When Int $C$ is not empty, we can define

$$
x \gg y \Leftrightarrow x-y \in \operatorname{Int} C .
$$

Definition 2 The dual cone of $C$ is defined as

$$
C^{*}=\left\{\lambda \in\left(\mathbb{R}^{N}\right)^{*} \mid \lambda(C) \geq 0\right\} .
$$

An immediate consequence is

$$
\begin{aligned}
x \in C & \Leftrightarrow \lambda(x) \geq 0, \forall \lambda \in C^{*} \\
x \in \operatorname{Int} C & \Leftrightarrow \lambda(x)>0, \forall \lambda \in C^{*} \backslash\{0\} .
\end{aligned}
$$

With this partial ordering on $\mathbb{R}^{N}$, we analyze certain features of the dynamics of an ordinary differential equation:

$$
\frac{d z}{d t}=F(z)
$$

where $F: \mathbb{R}^{N} \rightarrow \mathbb{R}^{N}$ is a $C^{1}$ vector field. We are interested in a special class of equations which preserve the ordering along the trajectories. For simplicity, the solutions of (66) are assumed to exist for all $t \geq 0$ in the sets considered below.

Definition 3 The flow $\phi_{t}$ of (6) is said to have (eventually) positive derivatives on a set $V \subseteq \mathbb{R}^{N}$, if $\left[D_{z} \phi_{t}(z)\right] x \in$ Int $C$ for all $x \in C \backslash\{0\}, z \in V$, and $t \geq 0$ ( $t \geq t_{0}$ for some $\left.t_{0}>0\right)$.

It is worth noticing that $\left[D_{z} \phi_{t}(z)\right] x \in \operatorname{Int} C$ is equivalent to $\lambda\left(\left[D_{z} \phi_{t}(z)\right] x\right)>0$ for all $\lambda \in C^{*}$ with norm one. We will use this fact in the proof of the next lemma, which deals with "regular" perturbations in the dynamics. The proof is in the same spirit as in Theorem 1.2 of [18], but generalized to the arbitrary cone $C$.

Lemma 1 Assume $V \subset \mathbb{R}^{N}$ is a compact set in which the flow $\phi_{t}$ of (마) has eventually positive derivatives. Then there exists $\delta>0$ with the following property. Let $\psi_{t}$ denote the flow of a $C^{1}$ vector field $G$ such that the $C^{1}$ norm of $F(z)-G(z)$ is less than $\delta$ for all $z$ in $V$. Then there exists $t_{*}>0$ such that if $\psi_{s}(z) \in V$ for all $s \in[0, t]$ where $t \geq t_{*}$, then $\left[D_{z} \psi_{t}(z)\right] x \in \operatorname{Int} C$ for all $z \in V$ and $x \in C \backslash\{0\}$. 
Proof. Pick $t_{0}>0$ so that $\lambda\left(\left[D_{z} \phi_{t}(z)\right] x\right)>0$ for all $t \geq t_{0}, z \in V, \lambda \in C^{*}, x \in C$ with $|\lambda|=1,|x|=1$. Then there exists $\delta>0$ with the property that when the $C^{1}$ norm of $F(z)-G(z)$ is less than $\delta$, we have $\lambda\left(\left[D_{z} \psi_{t}(z)\right] x\right)>0$ for $t_{0} \leq t \leq 2 t_{0}$.

When $t>2 t_{0}$, we write $t=r+k t_{0}$, where $t_{0} \leq r<2 t_{0}$ and $k \in \mathbb{N}$. If $\psi_{s}(z) \in V$ for all $s \in[0, t]$, we can define $z_{j}:=\psi_{j t_{0}}(z)$ for $j=0, \ldots, k$. For any $x \in C \backslash\{0\}$, using the chain rule, we have:

$$
\left[D_{z} \psi_{t}(z)\right] x=\left[D_{z} \psi_{r}\left(z_{k}\right)\right]\left[D_{z} \psi_{t_{0}}\left(z_{k-1}\right)\right] \cdots\left[D_{z} \psi_{t_{0}}\left(z_{0}\right)\right] x
$$

By induction, it is easy to see that $\left[D_{z} \psi_{t}(z)\right] x \in \operatorname{Int} C$.

Corollary 1 If $V$ is positively invariant under the flow $\psi_{t}$, then $\psi_{t}$ has eventually positive derivatives in $V$.

Proof. If $V$ is positively invariant under the flow $\psi_{t}$, then for any $z \in V$ the condition $\psi_{s}(z) \in V$ for $s \in[0, t]$ is satisfied for all $t \geq 0$. By the previous lemma, $\psi_{t}$ has eventually positive derivatives in $V$.

Definition 4 The system (마) or the flow $\phi_{t}$ of (6) is called monotone (resp. strongly monotone) in a set $W \subseteq \mathbb{R}^{N}$, if for all $t \geq 0$ and $z_{1}, z_{2} \in W$,

$$
\begin{aligned}
z_{1} \geq z_{2} \Rightarrow & \phi_{t}\left(z_{1}\right) \geq \phi_{t}\left(z_{2}\right) \\
& \left(\operatorname{resp} . \phi_{t}\left(z_{1}\right) \gg \phi_{t}\left(z_{2}\right) \text { when } z_{1} \neq z_{2}\right) .
\end{aligned}
$$

It is eventually (strongly) monotone if there exists $t_{0} \geq 0$ such that $\phi_{t}$ is (strongly) monotone for all $t \geq t_{0}$.

Definition 5 An set $W \subseteq \mathbb{R}^{N}$ is called p-convex, if $W$ contains the entire line segment joining $x$ and $y$ whenever $x \leq y, x, y \in W$.

The next two propositions discuss the relations between the two definitions, (eventually) positive derivatives and (eventually) strongly monotone.

Proposition 1 Let $W \subseteq \mathbb{R}^{N}$ be $p$-convex. If the flow $\phi_{t}$ has (eventually) positive derivatives in $W$, then it is (eventually) strongly monotone in $W$.

Proof. For any $z_{1}>z_{2} \in W, \lambda \in C^{*} \backslash\{0\}$ and $t \geq 0\left(t \geq t_{0}\right.$ for some $\left.t_{0}>0\right)$, we have that $\lambda\left(\phi_{t}\left(z_{1}\right)-\phi_{t}\left(z_{2}\right)\right)$ equals

$$
\int_{0}^{1} \lambda\left(\left[D_{z} \phi_{t}\left(s z_{1}+(1-s) z_{2}\right)\right]\left(z_{1}-z_{2}\right)\right) d s>0
$$

Therefore, $\phi_{t}$ is (eventually) strongly monotone in $W$.

Proposition 2 Suppose $\phi_{t}$ is (eventually) strongly monotone on an open set $U \subseteq \mathbb{R}^{N}$. Then $\phi_{t}$ has (eventually) positive derivatives in $U$.

Proof. Fix $t>0$ such that $\phi_{t}$ as a function from $U$ to $\mathbb{R}^{N}$ is strongly monotone (i.e. $\phi_{t}\left(z_{1}\right) \gg \phi_{t}\left(z_{2}\right)$, whenever $z_{1} \geq z_{2}, z_{1} \neq z_{2}$ in $\left.U\right)$.

For any $\lambda \in C^{*} \backslash\{0\}, x \in C \backslash\{0\}$, and $z \in U$,

$$
\lambda\left(\left[D_{z} \phi_{t}(z)\right] x\right)=\lim _{h \rightarrow 0} h^{-1} \lambda\left(\phi_{t}(z+h x)-\phi_{t}(z)\right) .
$$

If $h>0$ is small enough such that $z+h x \in U$, then we have $\lambda\left(\phi_{t}(z+h x)-\phi_{t}(z)\right)>0$. Thus $\lambda\left(\left[D_{z} \phi_{t}(z)\right] x\right)>$ 0 , and $\left[D_{z} \phi_{t}(z)\right] x \in \operatorname{Int} C$. 
Lemma 2 Suppose that the flow $\phi_{t}$ of (6) has compact closure and eventually positive derivatives in a p-convex set $W \subseteq \mathbb{R}^{N}$. If the set of equilibria is totally disconnected (e.g. countable), then the forward trajectory starting from almost every point in $W$ converges to an equilibrium.

Proof. By Proposition 1, $\phi_{t}$ is eventually strongly monotone. The result easily follows from Hirsch's Generic Convergence Theorem ([19], [37]).

\section{Details of the Proof}

Our approach to solve the varying domain problem is motivated by Nipp [30]. The idea is to extend the vector field from $U \times V \times[0, \varepsilon]$ to $\mathbb{R}^{n} \times \mathbb{R}^{m} \times\left[0, \varepsilon_{0}\right]$, then apply geometric singular perturbation theorems (33]) on $\mathbb{R}^{n} \times \mathbb{R}^{m} \times\left[0, \varepsilon_{0}\right]$, and finally restrict the flows to $D_{\varepsilon}$ for the generic convergence result.

\subsection{Extensions of the vector field}

For a given compact set $K \subset \mathbb{R}^{n}\left(K_{0} \subseteq K \subset U\right)$, the following procedure is adopted from [30] to extend a $C_{b}^{r}$ function with respect to the $x$ coordinate from $U$ to $\mathbb{R}^{n}$, such that the extended function is $C_{b}^{r}$ and agrees with the old one on $K$. This is a routine "smooth patching" argument.

Let $U_{1}$ be an open subset of $U$ with $C^{r}$ boundary and such that $K \subset U_{1} \subseteq U$. For $\Theta_{0}>0$ sufficiently small, define

$$
U_{1}^{\Theta_{0}}:=\left\{x \in U_{1} \mid \Theta(x) \geq \Theta_{0}\right\}, \text { where } \Theta(x):=\min _{u \in \partial U_{1}}|x-u|,
$$

such that $K$ is contained in $U_{1}^{\Theta_{0}}$. Consider the scalar $C^{\infty}$ function $\rho$ :

$$
\rho(a):= \begin{cases}0 & a \leq 0 \\ \exp (1-\exp (a-1) / a) & 0<a<1 \\ 1 & a \geq 1\end{cases}
$$

Define

$$
\hat{\Theta}(x):= \begin{cases}0 & x \in \mathbb{R}^{n} \backslash U_{1} \\ \Theta(x) & x \in U_{1} \backslash U_{1}^{\Theta_{0}} \\ \Theta_{0} & x \in U_{1}^{\Theta_{0}}\end{cases}
$$

and

$$
\bar{\Theta}(x):=\rho\left(\frac{\hat{\Theta}(x)}{\Theta_{0}}\right) .
$$

For any $q \in C_{b}^{r}(U)$, let

$$
\overline{\bar{q}}(x):=\left\{\begin{array}{ll}
q(x) & x \in U_{1} \\
0 & x \in \mathbb{R}^{n} \backslash U_{1},
\end{array} \text { and } \bar{q}(x):=\bar{\Theta}(x) \overline{\bar{q}}(x) .\right.
$$

Then $\bar{q}(x) \in C_{b}^{r}\left(\mathbb{R}^{n}\right)$ and $\bar{q}(x) \equiv q(x)$ on $K$.

We fix some $d_{0}>0$ such that

$$
D_{d_{0}}:=\left\{z \in \mathbb{R}^{m}|| z \mid \leq d_{0}\right\} \subset \bigcap_{x \in K}\left\{z \mid z+m_{0}(x) \in V\right\} .
$$


Then we extend the functions $f_{1}$ and $m_{0}$ to $\bar{f}_{1}$ and $\bar{m}_{0}$ respectively with respect to $x$ in the above way. To extend $g_{1}$, let us first rewrite the differential equation for $z$ as:

$$
\frac{d z}{d \tau}=[B(x)+C(x, z)] z+\varepsilon H(x, z, \varepsilon)-\varepsilon\left[D_{x} m_{0}(x)\right] f_{1}(x, z, \varepsilon),
$$

where

$$
B(x)=D_{y} g_{0}\left(x, m_{0}(x), 0\right) \text { and } C(x, 0)=0 .
$$

Following the above procedures, we extend the functions $C$ and $H$ to $\bar{C}$ and $\bar{H}$, but the extension of $B$ is defined as

$$
\bar{B}(x):=\bar{\Theta}(x) \overline{\bar{B}}(x)-\mu(1-\bar{\Theta}(x)) I_{n},
$$

where $\mu$ is the positive constant such that the real parts of all eigenvalues of $B(x)$ is less than $-\mu$ for every $x \in K$. According to the definition of $\bar{B}(x)$, all eigenvalues of $\bar{B}(x)$ will have negative real parts less than $-\mu$ for every $x \in \mathbb{R}^{n}$. The extension $\bar{g}_{1}$, defined as:

$$
[\bar{B}(x)+\bar{C}(x, z)] z+\varepsilon \bar{H}(x, z, \varepsilon)-\varepsilon\left[D_{x} \bar{m}_{0}(x)\right] \bar{f}_{1}(x, z, \varepsilon),
$$

is then $C_{b}^{r-1}\left(\mathbb{R}^{n} \times D_{d_{0}} \times\left[0, \varepsilon_{0}\right]\right)$ and agrees with $g_{1}$ on $K \times D_{d_{0}} \times\left[0, \varepsilon_{0}\right]$.

To extend functions $\bar{f}_{1}$ and $\bar{g}_{1}$ in the $z$ direction from $D_{d_{0}}$ to $\mathbb{R}^{m}$, we use the same extension technique but with respect to $z$. Let us denote the extensions of $\bar{f}_{1}, \bar{C}, \bar{H}$ and the function $z=z$ by $\tilde{f}_{1}, \tilde{C}, \tilde{H}$ and $\tilde{z}$ respectively, then define $\tilde{g}_{1}$ as:

$$
[\bar{B}(x)+\tilde{C}(x, z)] \tilde{z}(z)+\varepsilon \tilde{H}(x, z, \varepsilon)-\varepsilon\left[D_{x} \bar{m}_{0}(x)\right] \tilde{f}_{1}(x, z, \varepsilon),
$$

which is now $C_{b}^{r-1}\left(\mathbb{R}^{n} \times \mathbb{R}^{m} \times\left[0, \varepsilon_{0}\right]\right)$ and agrees with $g_{1}$ on $K \times D_{d_{1}} \times\left[0, \varepsilon_{0}\right]$ for some $d_{1}$ slightly less than $d_{0}$. Notice that $z=0$ is a solution of $\tilde{g}_{1}(x, z, 0)=0$, which guarantees that for the extended system in $(x, y)$ coordinates $\left(y=z+\bar{m}_{0}(x)\right)$ :

$$
\begin{aligned}
& \frac{d x}{d \tau}=\varepsilon f(x, y, \varepsilon) \\
& \frac{d y}{d \tau}=g(x, y, \varepsilon),
\end{aligned}
$$

$y=\bar{m}_{0}(x)$ is the solution of $g(x, y, 0)=0$. To summarize, (7) satisfies

E1 The functions

$$
\begin{gathered}
f \in C_{b}^{r}\left(\mathbb{R}^{n} \times \mathbb{R}^{m} \times\left[0, \varepsilon_{0}\right]\right), \\
g \in C_{b}^{r-1}\left(\mathbb{R}^{n} \times \mathbb{R}^{m} \times\left[0, \varepsilon_{0}\right]\right), \\
\bar{m}_{0} \in C_{b}^{r}\left(\mathbb{R}^{n}\right), \quad g\left(x, \bar{m}_{0}(x), 0\right)=0, \quad \forall x \in \mathbb{R}^{n} .
\end{gathered}
$$

E2 All eigenvalues of the matrix $D_{y} g\left(x, \bar{m}_{0}(x), 0\right)$ have negative real parts less than $-\mu$ for every $x \in \mathbb{R}^{n}$.

E3 The function $\bar{m}_{0}$ coincides with $m_{0}$ on $K$, and the functions $f$ and $g$ coincide with $f_{0}$ and $g_{0}$ respectively on

$$
\Omega_{d_{1}}:=\left\{(x, y)|x \in K,| y-m_{0}(x) \mid \leq d_{1}\right\}
$$

Conditions E1 and E2 are the assumptions for geometric singular perturbation theorems, and condition E3 ensures that on $\Omega_{d_{1}}$ the flow of (2) coincides with the flow of (7). If we apply geometric singular perturbation theorems to (7) on $\mathbb{R}^{n} \times \mathbb{R}^{m} \times\left[0, \varepsilon_{0}\right]$, the exact same results are true for (2) on $\Omega_{d_{1}}$. For the rest of the paper, we identify the flow of (7) and the flow of (2) on $\Omega_{d_{1}}$ without further mentioning this fact.(Later, in Lemmas 447, when globalizing the results, we consider again the original system.) 


\subsection{Geometric singular perturbation theory}

The theory of geometric singular perturbation can be traced back to the work of Fenichel [12, which first revealed the geometric aspects of singular perturbation problems. Later on, the works by Knobloch and Aulbach [25], Nipp [30], and Sakamoto [33] also presented results similar to [12]. By now, the theory is fairly standard, and there have been enormous applications to traveling waves of partial differential equations, see [23] and the references there. For control theoretic applications, see [1, 21].

To apply geometric singular perturbation theorems to the vector field on $\mathbb{R}^{n} \times \mathbb{R}^{m} \times\left[0, \varepsilon_{0}\right]$, we use the theorems stated in [33]. The following lemma is a restatement of the theorems in [33], and we refer to [33] for the proof.

Lemma 3 Under conditions $\mathbf{E 1}$ and $\mathbf{E 2}$, there exists a positive $\varepsilon_{1}<\varepsilon_{0}$ such that for every $\varepsilon \in\left(0, \varepsilon_{1}\right]$ :

1. There is a $C_{b}^{r-1}$ function

$$
m: \mathbb{R}^{n} \times\left[0, \varepsilon_{1}\right] \rightarrow \mathbb{R}^{m}
$$

such that the set $M_{\varepsilon}$ defined by

$$
M_{\varepsilon}:=\left\{(x, m(x, \varepsilon)) \mid x \in \mathbb{R}^{n}\right\}
$$

is invariant under the flow generated by (7). Moreover,

$$
\sup _{x \in \mathbb{R}^{n}}\left|m(x, \varepsilon)-\bar{m}_{0}(x)\right|=O(\varepsilon) \text {, as } \varepsilon \rightarrow 0 .
$$

In particular, we have $m(x, 0)=\bar{m}_{0}(x)$ for all $x \in \mathbb{R}^{n}$.

2. The set consisting of all the points $\left(x_{0}, y_{0}\right)$ such that

$$
\sup _{\tau \geq 0}\left|y\left(\tau ; x_{0}, y_{0}\right)-m\left(x\left(\tau ; x_{0}, y_{0}\right), \varepsilon\right)\right| e^{\frac{\mu \tau}{4}}<\infty
$$

where $\left(x\left(\tau ; x_{0}, y_{0}\right), y\left(\tau ; x_{0}, y_{0}\right)\right)$ is the solution of (7) passing through $\left(x_{0}, y_{0}\right)$ at $\tau=0$, is a $C^{r-1}$ immersed submanifold in $\mathbb{R}^{n} \times \mathbb{R}^{m}$ of dimension $n+m$, denoted by $W^{s}\left(M_{\varepsilon}\right)$.

3. There is a positive constant $\delta_{0}$ such that if

$$
\sup _{\tau \geq 0}\left|y\left(\tau ; x_{0}, y_{0}\right)-m\left(x\left(\tau ; x_{0}, y_{0}\right), \varepsilon\right)\right|<\delta_{0},
$$

then $\left(x_{0}, y_{0}\right) \in W^{s}\left(M_{\varepsilon}\right)$.

4. The manifold $W^{s}\left(M_{\varepsilon}\right)$ is a disjoint union of $C^{r-1}$-immersed manifolds $W_{\varepsilon}^{s}(\xi)$ of dimension $m$ :

$$
W^{s}\left(M_{\varepsilon}\right)=\bigcup_{\xi \in \mathbb{R}^{n}} W_{\varepsilon}^{s}(\xi)
$$

For each $\xi \in \mathbb{R}^{n}$, let $H_{\varepsilon}(\xi)(\tau)$ be the solution for $\tau \geq 0$ of

$$
\frac{d x}{d \tau}=\varepsilon f(x, m(x, \varepsilon), \varepsilon), \quad x(0)=\xi \in \mathbb{R}^{n} .
$$

Then, the manifold $W_{\varepsilon}^{s}(\xi)$ is the set

$$
\left\{\left(x_{0}, y_{0}\right)\left|\sup _{\tau \geq 0}\right| \tilde{x}(\tau)\left|e^{\frac{\mu \tau}{4}}<\infty, \sup _{\tau \geq 0}\right| \tilde{y}(\tau) \mid e^{\frac{\mu \tau}{4}}<\infty\right\},
$$


where

$$
\begin{aligned}
& \tilde{x}(\tau)=x\left(\tau ; x_{0}, y_{0}\right)-H_{\varepsilon}(\xi)(\tau), \\
& \tilde{y}(\tau)=y\left(\tau ; x_{0}, y_{0}\right)-m\left(H_{\varepsilon}(\xi)(\tau), \varepsilon\right) .
\end{aligned}
$$

5. The fibers are "positively invariant" in the sense that $W_{\varepsilon}^{s}\left(H_{\varepsilon}(\xi)(\tau)\right)$ is the set

$$
\left\{\left(x\left(\tau ; x_{0}, y_{0}\right), y\left(\tau ; x_{0}, y_{0}\right)\right) \mid\left(x_{0}, y_{0}\right) \in W_{\varepsilon}^{s}(\xi)\right\}
$$

for each $\tau \geq 0$, see Figure 2 .

6. The fibers restricted to the $\delta_{0}$ neighborhood of $M_{\varepsilon}$, denoted by $W_{\varepsilon, \delta_{0}}^{s}$, can be parametrized as follows. There are two $C_{b}^{r-1}$ functions

$$
\begin{gathered}
P_{\varepsilon, \delta_{0}}: \mathbb{R}^{n} \times D_{\delta_{0}} \rightarrow \mathbb{R}^{n} \\
Q_{\varepsilon, \delta_{0}}: \mathbb{R}^{m} \times D_{\delta_{0}} \rightarrow \mathbb{R}^{m},
\end{gathered}
$$

and a map

$$
T_{\varepsilon, \delta_{0}}: \mathbb{R}^{n} \times D_{\delta_{0}} \rightarrow \mathbb{R}^{n} \times \mathbb{R}^{m}
$$

mapping $(\xi, \eta)$ to $(x, y)$, where

$$
x=\xi+P_{\varepsilon, \delta_{0}}(\xi, \eta), \quad y=m(x, \varepsilon)+Q_{\varepsilon, \delta_{0}}(\xi, \eta)
$$

such that

$$
W_{\varepsilon, \delta_{0}}^{s}(\xi)=T_{\varepsilon, \delta_{0}}\left(\xi, D_{\delta_{0}}\right)
$$

Remark 2 The $\delta_{0}$ in property 3 can be chosen uniformly for $\varepsilon \in\left(0, \varepsilon_{0}\right]$. Without loss of generality, we assume that $\delta_{0}<d_{1}$.

Notice that property 4 insures that for each $\left(x_{0}, y_{0}\right) \in W^{s}\left(M_{\varepsilon}\right)$, there exists a $\xi$ such that

$$
\begin{gathered}
\left|x\left(\tau ; x_{0}, y_{0}\right)-H_{\varepsilon}(\xi)(\tau)\right| \rightarrow 0, \\
\left|y\left(\tau ; x_{0}, y_{0}\right)-m\left(H_{\varepsilon}(\xi)(\tau), \varepsilon\right)\right| \rightarrow 0 .
\end{gathered}
$$

as $t \rightarrow 0$. This is often referred as the "asymptotic phase" property, see Figure Q

\subsection{Further analysis of the dynamics}

The first property of Lemma 3 concludes the existence of an invariant manifold $M_{\varepsilon}$. There are two reasons to introduce $M_{\varepsilon}$. First, on $M_{\varepsilon}$ the $x$-equation is decoupled from the $y$-equation:

$$
\begin{aligned}
\frac{d x}{d t} & =f(x, m(x, \varepsilon), \varepsilon) \\
y(t) & =m(x(t), \varepsilon) .
\end{aligned}
$$

This reduction allows us to analyze a lower dimensional system, whose dynamics may have been well studied. Second, when $\varepsilon$ approaches zero, the limit of (8) is (5) on $K_{0}$. If (5) has some desirable property, it is natural to expect that this property is inherited by (8). An example of this principle is provided by the following Lemma: 


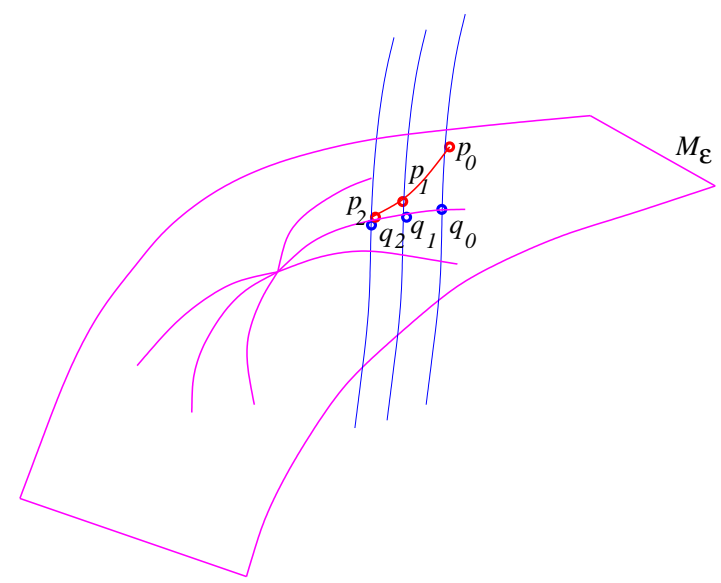

Figure 2: An illustration of the "positive invariant" and "asymptotic phase" properties. Let $p_{0}$ be a point on the fiber $W_{\varepsilon}^{s}\left(q_{0}\right)$ (vertical curve). Suppose the solution of (17) starting from $q_{0} \in M_{\varepsilon}$ evolves to $q_{1} \in M_{\varepsilon}$ at time $\tau_{1}$, then the solution of (77) starting from $p_{0}$ will evolve to $p_{1} \in W_{\varepsilon}^{s}\left(q_{1}\right)$ at time $\tau_{1}$. At time $\tau_{2}$, they evolve to $q_{2}, p_{2}$ respectively. These two solutions are always on the same fiber. If we know that the one starting from $q_{0}$ converges to a equilibrium, then the one starting from $p_{0}$ also converges to a equilibrium.

Lemma 4 There exists a positive constant $\varepsilon_{2}<\varepsilon_{1}$, such that for each $\varepsilon \in\left(0, \varepsilon_{2}\right)$, the flow $\psi_{t}^{\varepsilon}$ of (8) has eventually positive derivatives on $K_{\varepsilon}$, which is the projection of $M_{\varepsilon} \cap D_{\varepsilon}$ to the $x$-axis.

Proof. Assumption A6 states that the flow $\psi_{t}^{0}$ of the limiting system (5) has eventually positive derivatives on $K_{0}$. By the continuity of $m(x, \varepsilon)$ and $D_{\varepsilon}$ at $\varepsilon=0$, we can pick $\varepsilon_{2}$ small enough such that the flow $\psi_{t}^{0}$ has eventually positive derivatives on $K_{\varepsilon}$ for all $\varepsilon \in\left(0, \varepsilon_{2}\right)$. Applying Corollary 1, we conclude that the flow $\psi_{t}^{\varepsilon}$ of (8) has eventually positive derivatives on $K_{\varepsilon}$ provided $K_{\varepsilon}$ is positively invariant under (8), which follows easily from the facts that (7) is positively invariant on $D_{\varepsilon}$ and $M_{\varepsilon}$ is an invariant manifold.

The next Lemma asserts that the generic convergence property is preserved for (8), see Figure 3 ,

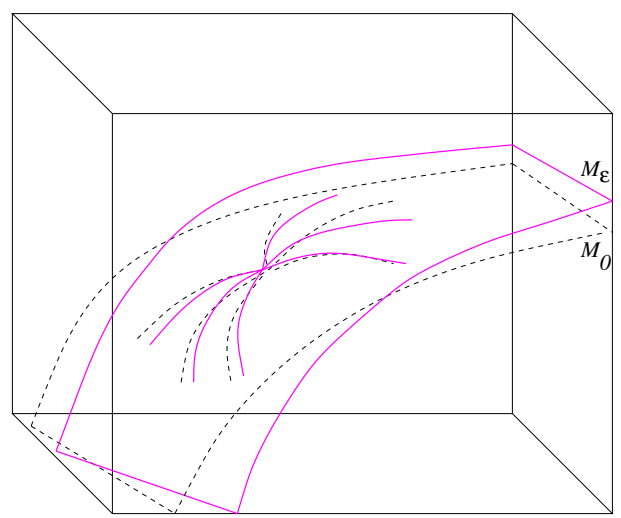

Figure 3: This is a sketch of the manifolds $M_{0}$ (surface bounded by dashed curves), $M_{\varepsilon}$ (surface bounded by dotted curves), and $D_{\varepsilon}$ (the cube). It highlights two major characters of $M_{\varepsilon}$. First, $M_{\varepsilon}$ is close to $M_{0}$. Second, the trajectories on $M_{\varepsilon}$ converge to equilibria if those on $M_{0}$ do.

Lemma 5 For each $\varepsilon \in\left(0, \varepsilon_{2}\right)$, there exists a set $C_{\varepsilon} \subseteq K_{\varepsilon}$ such that the forward trajectory of (8) starting from any point of $C_{\varepsilon}$ converges to some equilibrium, and the Lebesgue measure of $K_{\varepsilon} \backslash C_{\varepsilon}$ is zero. 
Proof. Apply Lemma 2 and Lemma 4 under assumptions A5 and A7.

By now, we have discussed flows restricted to the invariant manifold $M_{\varepsilon}$. Next, we will explore the conditions for a point to be on $W^{s}\left(M_{\varepsilon}\right)$, the stable manifold of $M_{\varepsilon}$. Property 3 of Lemma 3 provides a sufficient condition, namely, any point $\left(x_{0}, y_{0}\right)$ such that

$$
\sup _{\tau \geq 0}\left|y\left(\tau ; x_{0}, y_{0}\right)-m\left(x\left(\tau ; x_{0}, y_{0}\right), \varepsilon\right)\right|<\delta_{0}
$$

is on $W^{s}\left(M_{\varepsilon}\right)$. In fact, if we know that the difference between $y_{0}$ and $m\left(x_{0}, \varepsilon\right)$ is sufficiently small, then the above condition is always satisfied. More precisely, we have:

Lemma 6 There exists $\varepsilon_{3}>0, \delta_{0}>d>0$, such that for each $\varepsilon \in\left(0, \varepsilon_{3}\right)$, if the initial condition satisfies $\left|y_{0}-m\left(x_{0}, \varepsilon\right)\right|<d$, then (9) holds, i.e. $\left(x_{0}, y_{0}\right) \in W^{s}\left(M_{\varepsilon}\right)$.

Proof. Follows from the proof of Claim 1 in [30].

Before we get further into the technical details, let us give an outline of the proof of the main theorem. The proof can be decomposed into three steps. First, we show that almost every trajectory on $D_{\varepsilon} \bigcap M_{\varepsilon}$ converges to some equilibrium. This is precisely Lemma 5. Second, we show that almost every trajectory starting from $W^{s}\left(M_{\varepsilon}\right)$ converges to some equilibrium. This follows from Lemma 5 and the "asymptotic phase" property in Lemma 3, but we still need to show that the set of non-convergent initial conditions is of measure zero. The last step is to show that all trajectories in $D_{\varepsilon}$ will eventually stay in $W^{s}\left(M_{\varepsilon}\right)$, which is our next lemma:

Lemma 7 There exist positive $\tau_{0}$ and $\varepsilon_{4}<\varepsilon_{3}$, such that $\left(x\left(\tau_{0}\right), y\left(\tau_{0}\right)\right) \in W^{s}\left(M_{\varepsilon}\right)$ for all $\varepsilon \in\left(0, \varepsilon_{4}\right)$, where $(x(\tau), y(\tau))$ is the solution to (2) with the initial condition $\left(x_{0}, y_{0}\right) \in D_{\varepsilon}$.

Proof. It is convenient to consider the problem in $(x, z)$ coordinates. Let $(x(\tau), z(\tau))$ be the solution to (3) with initial condition $\left(x_{0}, z_{0}\right)$, where $z_{0}=y_{0}-m\left(x_{0}, 0\right)$. We first show that there exists a $\tau_{0}$ such that $\left|z\left(\tau_{0}\right)\right| \leq d / 2$.

Expanding $g_{1}(x, z, \varepsilon)$ at the point $\left(x_{0}, z, 0\right)$, the equation of $z$ becomes

$$
\frac{d z}{d \tau}=g_{1}\left(x_{0}, z, 0\right)+\frac{\partial g_{1}}{\partial x}(\xi, z, 0)\left(x-x_{0}\right)+\varepsilon R(x, z, \varepsilon)
$$

for some $\xi(\tau)$ between $x_{0}$ and $x(\tau)$ (where $\xi(\tau)$ can be picked continuously in $\tau$ ). Let us write

$$
z(\tau)=z^{0}(\tau)+w(\tau)
$$

where $z^{0}(\tau)$ is the solution to (4) with initial the condition $z^{0}(0)=z_{0}$, and $w(\tau)$ satisfies

$$
\begin{aligned}
\frac{d w}{d \tau} & =g_{1}\left(x_{0}, z, 0\right)-g_{1}\left(x_{0}, z^{0}, 0\right)+\frac{\partial g_{1}}{\partial x}(\xi, z, 0)\left(x-x_{0}\right)+\varepsilon R(x, z, \varepsilon) \\
& =\frac{\partial g_{1}}{\partial z}\left(x_{0}, \zeta, 0\right) w+\varepsilon \frac{\partial g_{1}}{\partial x}(\xi, z, 0) \int_{0}^{\tau} f_{1}(x(s), z(s), \varepsilon) d s+\varepsilon R(x, z, \varepsilon),
\end{aligned}
$$

with the initial condition $w(0)=0$ and some $\zeta(\tau)$ between $z^{0}(\tau)$ and $z(\tau)$ (where $\zeta(\tau)$ can be picked continuously in $\tau$ ).

By assumption A3, there exist a positive $\tau_{0}$ such that $\left|z^{0}(\tau)\right| \leq d / 4$ for all $\tau \geq \tau_{0}$. Notice that we are working on the compact set $D_{\varepsilon}$, so $\tau_{0}$ can be chosen uniformly for all initial conditions in $D_{\varepsilon}$. 
We write the solution of (10) as:

$$
w(\tau)=\int_{0}^{\tau} \frac{\partial g_{1}}{\partial z}\left(x_{0}, \zeta, 0\right) w d s+\varepsilon \int_{0}^{\tau}\left(\frac{\partial g_{1}}{\partial x}(\xi, z, 0) \int_{0}^{s^{\prime}} f_{1}(x, z, \varepsilon) d s^{\prime}+R(x, z, \varepsilon)\right) d s
$$

Since the functions $f_{1}, R$ and the derivatives of $g_{1}$ are bounded on $D_{\varepsilon}$, we have:

$$
|w(\tau)| \leq \int_{0}^{\tau} L|w| d s+\varepsilon \int_{0}^{\tau}\left(M_{1} \int_{0}^{s^{\prime}} M_{2} d s^{\prime}+M_{3}\right) d s,
$$

for some positive constants $L, M_{i}, i=1,2,3$. The notation $|w|$ means the Euclidean norm of $w \in \mathbb{R}^{m}$. Moreover, if we define

$$
\alpha(\tau)=\int_{0}^{\tau}\left(M_{1} \int_{0}^{s^{\prime}} M_{2} d s^{\prime}+M_{3}\right) d s
$$

then

$$
|w(\tau)| \leq \int_{0}^{\tau} L|w| d s+\varepsilon \alpha\left(\tau_{0}\right),
$$

for all $\tau \in\left[0, \tau_{0}\right]$ as $\alpha$ is increasing in $\tau$. Applying Gronwall's inequality ([38]), we have:

$$
|w(\tau)| \leq \varepsilon \alpha\left(\tau_{0}\right) e^{L \tau}
$$

which holds in particular at $\tau=\tau_{0}$. Finally, we choose $\varepsilon_{4}$ small enough such that $\varepsilon \alpha\left(\tau_{0}\right) e^{L \tau_{0}}<d / 4$ and $|m(x, \varepsilon)-m(x, 0)|<d / 2$ for all $\varepsilon \in\left(0, \varepsilon_{4}\right)$. Then we have:

$$
\begin{aligned}
\left|y\left(\tau_{0}\right)-m\left(x\left(\tau_{0}\right), \varepsilon\right)\right| & \leq\left|y\left(\tau_{0}\right)-m\left(x\left(\tau_{0}\right), 0\right)\right|+\left|m\left(x\left(\tau_{0}\right), \varepsilon\right)-m\left(x\left(\tau_{0}\right), 0\right)\right| \\
& <\left|z\left(\tau_{0}\right)\right|+d / 2 \\
& <d / 2+d / 2=d
\end{aligned}
$$

That is, $\left(x\left(\tau_{0}\right), y\left(\tau_{0}\right)\right) \in W^{s}\left(M_{\varepsilon}\right)$ by Lemma 6 .

By now, we have completed all these three steps, and are ready to prove Theorem 1.

\subsection{Proof of Theorem 1}

Proof. Let $\varepsilon^{*}=\min \left\{\varepsilon_{2}, \varepsilon_{4}\right\}$. For $\varepsilon \in\left(0, \varepsilon^{*}\right)$, it is equivalent to prove the result for the fast system (2). Pick an arbitrary point $\left(x_{0}, y_{0}\right)$ in $D_{\varepsilon}$, and there are three cases:

1. $y_{0}=m\left(x_{0}, \varepsilon\right)$, that is, $\left(x_{0}, y_{0}\right) \in M_{\varepsilon} \bigcap D_{\varepsilon}$. By Lemma 5, the forward trajectory converges to an equilibrium except for a set of measure zero.

2. $0<\left|y_{0}-m\left(x_{0}, \varepsilon\right)\right|<d$. By Lemma 6, we know that $\left(x_{0}, y_{0}\right)$ is in $W^{s}\left(M_{\varepsilon}\right)$. Then, property 4 of Lemma 3 guarantees that the point $\left(x_{0}, y_{0}\right)$ is on some fiber $W_{\varepsilon, d}^{s}(\xi)$, where $\xi \in K_{\varepsilon}$. If $\xi \in C_{\varepsilon}$, that is, the forward trajectory of $\xi$ converges to some equilibrium, then by the "asymptotic phase" property of Lemma 3, the forward trajectory of $\left(x_{0}, y_{0}\right)$ also converges to an equilibrium. To deal with the case when $\xi$ is not in $C_{\varepsilon}$, it is enough to show that the set

$$
B_{\varepsilon, d}=\bigcup_{\xi \in K_{\varepsilon} \backslash C_{\varepsilon}} W_{\varepsilon, d}^{s}(\xi)
$$


has measure zero in $\mathbb{R}^{m+n}$. Define

$$
S_{\varepsilon, d}=\left(K_{\varepsilon} \backslash C_{\varepsilon}\right) \times D_{d}
$$

By Lemma 5, $K_{\varepsilon} \backslash C_{\varepsilon}$ has measure zero in $\mathbb{R}^{n}$, thus $S_{\varepsilon, d}$ has measure zero in $\mathbb{R}^{n} \times \mathbb{R}^{m}$. On the other hand, property 6 in Lemma 3 implies $B_{\varepsilon, d}=T_{\varepsilon, d}\left(S_{\varepsilon, d}\right)$. Since Lipschitz maps send measure zero sets to measure zero sets, $B_{\varepsilon, d}$ is of measure zero.

3. $\left|y_{0}-m\left(x_{0}, \varepsilon\right)\right| \geq d$. By Lemma 7 , the point $\left(x\left(\tau_{0}\right), y\left(\tau_{0}\right)\right)$ is in $W^{s}\left(M_{\varepsilon}\right)$ and we are back to case 2 . The proof is completed if the set $\phi_{-\tau_{0}}^{\varepsilon}\left(B_{\varepsilon, d}\right)$ has measure zero, where $\phi_{\tau}^{\varepsilon}$ is the flow of (2). This is true because $\phi_{\tau}^{\varepsilon}$ is a diffeomorphism for any finite $\tau$.

\section{Applications}

\subsection{An application to the dual futile cycle}

We assume that the reactions in Figure 1 follow the usual enzymatic mechanism ([13]):

$$
\begin{gathered}
S_{0}+E \underset{k_{-1}}{\stackrel{k_{1}}{\rightleftarrows}} C_{1} \stackrel{k_{2}}{\rightarrow} S_{1}+E \underset{k_{-3}}{\stackrel{k_{3}}{\rightleftarrows}} C_{2} \stackrel{k_{4}}{\rightarrow} S_{2}+E \\
S_{2}+F \underset{h_{-1}}{\stackrel{h_{1}}{\rightleftarrows}} C_{3} \stackrel{h_{2}}{\rightarrow} S_{1}+F \underset{h_{-3}}{\stackrel{h_{3}}{\rightleftarrows}} C_{4} \stackrel{h_{4}}{\rightarrow} S_{0}+F .
\end{gathered}
$$

There are three conservation relations:

$$
\begin{aligned}
S_{t o t} & =\left[S_{0}\right]+\left[S_{1}\right]+\left[S_{2}\right]+\left[C_{1}\right]+\left[C_{2}\right]+\left[C_{4}\right]+\left[C_{3}\right], \\
E_{t o t} & =[E]+\left[C_{1}\right]+\left[C_{2}\right], \\
F_{t o t} & =[F]+\left[C_{4}\right]+\left[C_{3}\right]
\end{aligned}
$$

where brackets indicate concentrations. Based on mass action kinetics, we have the following set of ordinary differential equations:

$$
\begin{aligned}
& \frac{d\left[S_{0}\right]}{d \tau}=h_{4}\left[C_{4}\right]-k_{1}\left[S_{0}\right][E]+k_{-1}\left[C_{1}\right] \\
& \frac{d\left[S_{2}\right]}{d \tau}=k_{4}\left[C_{2}\right]-h_{1}\left[S_{2}\right][F]+h_{-1}\left[C_{3}\right] \\
& \frac{d\left[C_{1}\right]}{d \tau}=k_{1}\left[S_{0}\right][E]-\left(k_{-1}+k_{2}\right)\left[C_{1}\right] \\
& \frac{d\left[C_{2}\right]}{d \tau}=k_{3}\left[S_{1}\right][E]-\left(k_{-3}+k_{4}\right)\left[C_{2}\right] \\
& \frac{d\left[C_{4}\right]}{d \tau}=h_{3}\left[S_{1}\right][F]-\left(h_{-3}+h_{4}\right)\left[C_{4}\right] \\
& \frac{d\left[C_{3}\right]}{d \tau}=h_{1}\left[S_{2}\right][F]-\left(h_{-1}+h_{2}\right)\left[C_{3}\right] .
\end{aligned}
$$


After rescaling the concentrations and time, (11) becomes:

$$
\begin{aligned}
\frac{d x_{1}}{d t} & =-k_{1} S_{t o t} x_{1}\left(1-y_{1}-y_{2}\right)+k_{-1} y_{1}+h_{4} c y_{3} \\
\frac{d x_{2}}{d t} & =-h_{1} S_{t o t} c x_{2}\left(1-y_{3}-y_{4}\right)+h_{-1} c y_{4}+k_{4} y_{2} \\
\varepsilon \frac{d y_{1}}{d t} & =k_{1} S_{t o t} x_{1}\left(1-y_{1}-y_{2}\right)-\left(k_{-1}+k_{2}\right) y_{1} \\
\varepsilon \frac{d y_{2}}{d t} & =k_{3} S_{t o t}\left(1-x_{1}-x_{2}-\varepsilon y_{1}-\varepsilon y_{2}-\varepsilon c y_{3}-\varepsilon c y_{4}\right) \times\left(1-y_{1}-y_{2}\right)-\left(k_{-3}+k_{4}\right) y_{2} \\
\varepsilon \frac{d y_{3}}{d t} & =h_{3} S_{t o t}\left(1-x_{1}-x_{2}-\varepsilon y_{1}-\varepsilon y_{2}-\varepsilon c y_{3}-\varepsilon c y_{4}\right) \times\left(1-y_{3}-y_{4}\right)-\left(h_{-3}+h_{4}\right) y_{3} \\
\varepsilon \frac{d y_{4}}{d t} & =h_{1} S_{t o t} x_{2}\left(1-y_{3}-y_{4}\right)-\left(h_{-1}+h_{2}\right) y_{4},
\end{aligned}
$$

where

$$
\begin{gathered}
x_{1}=\frac{\left[S_{0}\right]}{S_{t o t}}, \quad x_{2}=\frac{\left[S_{2}\right]}{S_{t o t}}, \quad y_{1}=\frac{\left[C_{1}\right]}{E_{t o t}}, \quad y_{2}=\frac{\left[C_{2}\right]}{E_{t o t}}, \\
y_{3}=\frac{\left[C_{4}\right]}{F_{t o t}}, \quad y_{4}=\frac{\left[C_{3}\right]}{F_{t o t}}, \quad \varepsilon=\frac{E_{t o t}}{S_{t o t}}, \quad c=\frac{F_{t o t}}{E_{t o t}}, \quad t=\tau \varepsilon .
\end{gathered}
$$

These equations are in the form of (1). The conservation laws suggest taking $\varepsilon_{0}=1 /(1+c)$ and

$$
\begin{aligned}
D_{\varepsilon}= & \left\{\left(x_{1}, x_{2}, y_{1}, y_{2}, y_{3}, y_{4}\right) \mid 0 \leq y_{1}+y_{2} \leq 1,0 \leq y_{3}+y_{4} \leq 1,\right. \\
& \left.x_{1} \geq 0, x_{2} \geq 0,0 \leq x_{1}+x_{2}+\varepsilon\left(y_{1}+y_{2}+c y_{3}+c y_{4}\right) \leq 1\right\} .
\end{aligned}
$$

For $\varepsilon \in\left(0, \varepsilon_{0}\right]$, taking the inner product of the normal of $\partial D_{\varepsilon}$ and the vector field, it is easy to check that (12) is positively invariant on $D_{\varepsilon}$, so $\mathbf{A 5}$ holds. We want to emphasize that in this example the domain $D_{\varepsilon}$ is a convex polytope varying with $\varepsilon$.

It can be proved that on $D_{\epsilon}$ system (12) has at most a finite number of steady states, and thus $\mathbf{A} 7$ holds. This is a consequence of a more general result, proved using some of the ideas given in [16], concerning the number of steady states of more general systems of phosphorylation/dephosphorylation reactions, see [45].

Solving $g_{0}(x, y, 0)=0$, we get

$$
\begin{aligned}
& y_{1}=\frac{x_{1}}{\frac{K_{m 1}}{S_{t o t}}+\frac{K_{m 1}\left(1-x_{1}-x_{2}\right)}{K_{m 2}}+x_{1}}, \\
& y_{2}=\frac{\frac{K_{m 1}\left(1-x_{1}-x_{2}\right)}{K_{m 2}}}{\frac{K_{m 1}}{S_{t o t}}+\frac{K_{m 1}\left(1-x_{1}-x_{2}\right)}{K_{m 2}}+x_{1}}, \\
& y_{3}=\frac{\frac{K_{m 3}\left(1-x_{1}-x_{2}\right)}{K_{m 4}}}{\frac{K_{m 3}}{S_{t o t}}+\frac{K_{m 3}\left(1-x_{1}-x_{2}\right)}{K_{m 4}}+x_{2}}, \\
& y_{4}=\frac{x_{2}}{\frac{K_{m 3}}{S_{t o t}}+\frac{K_{m 3}\left(1-x_{1}-x_{2}\right)}{K_{m 4}}+x_{2}},
\end{aligned}
$$

where $K_{m 1}, K_{m 2}, K_{m 3}$ and $K_{m 4}$ are the Michaelis-Menten constants defined as

$$
K_{m 1}=\frac{k_{-1}+k_{2}}{k_{1}}, \quad K_{m 2}=\frac{k_{-3}+k_{4}}{k_{3}}, \quad K_{m 3}=\frac{h_{-1}+h_{2}}{h_{1}}, \quad K_{m 4}=\frac{h_{-3}+h_{4}}{h_{3}} .
$$


Now, we need to find a proper set $U \subset \mathbb{R}^{2}$ satisfying assumptions A1-A4. Suppose that $U$ has the form

$$
U=\left\{\left(x_{1}, x_{2}\right) \mid x_{1}>-\sigma, x_{2}>-\sigma, x_{1}+x_{2}<1+\sigma\right\},
$$

for some positive $\sigma$, and $V$ is any bounded open set such that $D_{\varepsilon}$ is contained in $U \times V$, then $\mathbf{A} \mathbf{1}$ follows naturally. Moreover, if

$$
\sigma \leq \sigma_{0}:=\min \left\{\frac{K_{m 1} K_{m 2}}{S_{t o t}\left(K_{m 1}+K_{m 2}\right)}, \frac{K_{m 3} K_{m 4}}{S_{t o t}\left(K_{m 3}+K_{m 4}\right)}\right\}
$$

A2 also holds. To check A4, let us look at the matrix:

$$
B(x):=D_{y} g_{0}\left(x, m_{0}(x), 0\right)=\left(\begin{array}{cc}
B_{1}(x) & 0 \\
0 & B_{2}(x)
\end{array}\right),
$$

where

$$
B_{1}(x)=\left(\begin{array}{cc}
-k_{1} S_{t o t} x_{1}-\left(k_{-1}+k_{2}\right) & -k_{1} S_{t o t} x_{1} \\
-k_{3} S_{t o t}\left(1-x_{1}-x_{2}\right) & -k_{3} S_{t o t}\left(1-x_{1}-x_{2}\right)-\left(k_{-3}+k_{4}\right)
\end{array}\right)
$$

and

$$
B_{2}(x)=\left(\begin{array}{cc}
-h_{3} S_{t o t}\left(1-x_{1}-x_{2}\right)-\left(h_{-3}+h_{4}\right) & -h_{3} S_{t o t}\left(1-x_{1}-x_{2}\right) \\
-h_{1} S_{t o t} x_{2} & -h_{1} S_{t o t} x_{2}-\left(h_{-1}+h_{2}\right.
\end{array}\right) .
$$

If both matrices $B_{1}$ and $B_{2}$ have negative traces and positive determinants, then $\mathbf{A} 4$ holds.

Let us consider $B_{1}$ first. The trace of $B_{1}$ is

$$
-k_{1} S_{t o t} x_{1}-\left(k_{-1}+k_{2}\right)-k_{3} S_{t o t}\left(1-x_{1}-x_{2}\right)-\left(k_{-3}+k_{4}\right)
$$

It is negative provided that

$$
\sigma \leq \frac{k_{-1}+k_{2}+k_{-3}+k_{4}}{S_{t o t}\left(k_{1}+k_{3}\right)}
$$

The determinant of $B_{1}$ is

$$
k_{1}\left(k_{-3}+k_{4}\right) S_{t o t} x_{1}+k_{3}\left(k_{-1}+k_{2}\right) S_{t o t}\left(1-x_{1}-x_{2}\right)+\left(k_{-1}+k_{2}\right)\left(k_{-3}+k_{4}\right) .
$$

It is positive if

$$
\sigma \leq \frac{\left(k_{-1}+k_{2}\right)\left(k_{-3}+k_{4}\right)}{S_{t o t}\left(k_{1}\left(k_{-3}+k_{4}\right)+k_{3}\left(k_{-1}+k_{2}\right)\right)} .
$$

The condition for $B_{2}$ can be derived similarly. To summarize, if we take

$$
\begin{array}{r}
\sigma=\min \left\{\sigma_{0}, \frac{k_{-1}+k_{2}+k_{-3}+k_{4}}{S_{t o t}\left(k_{1}+k_{3}\right)}, \frac{\left(k_{-1}+k_{2}\right)\left(k_{-3}+k_{4}\right)}{S_{t o t}\left(k_{1}\left(k_{-3}+k_{4}\right)+k_{3}\left(k_{-1}+k_{2}\right)\right)},\right. \\
\left.\frac{h_{-1}+h_{2}+h_{-3}+h_{4}}{S_{t o t}\left(h_{1}+h_{3}\right)}, \frac{\left(h_{-1}+h_{2}\right)\left(h_{-3}+h_{4}\right)}{S_{t o t}\left(h_{1}\left(h_{-3}+h_{4}\right)+h_{3}\left(h_{-1}+h_{2}\right)\right)}\right\},
\end{array}
$$

then the assumptions A1, A2 and A4 will hold.

Notice that $\dot{y}$ in (12) is linear in $y$ when $\varepsilon=0$, so $g_{1}$ (defined as in (3)) is linear in $z$, and hence the equation for $z$ can be written as:

$$
\frac{d z}{d \tau}=B\left(x_{0}\right) z, \quad x_{0} \in U,
$$

where the matrix $B\left(x_{0}\right)$ is Hurwitz for every $x_{0} \in U$. Therefore, A3 also holds. 
To check A6, let us look at the reduced system $(\varepsilon=0$ in (12) $)$ :

$$
\begin{aligned}
& \frac{d x_{1}}{d t}=-\frac{k_{2} x_{1}}{\frac{K_{m 1}}{S_{t o t}}+\frac{K_{m 1}\left(1-x_{1}-x_{2}\right)}{K_{m 2}}+x_{1}}+\frac{h_{4} c \frac{K_{m 3}\left(1-x_{1}-x_{2}\right)}{K_{m 4}}}{\frac{K_{m 3}}{S_{t o t}}+\frac{K_{m 3}\left(1-x_{1}-x_{2}\right)}{K_{m 4}}+x_{2}}:=F_{1}\left(x_{1}, x_{2}\right) \\
& \frac{h_{2} c x_{2}}{d t}=-\frac{k_{4} \frac{K_{m 1}\left(1-x_{1}-x_{2}\right)}{K_{m 2}}}{\frac{K_{m 3}}{S_{t o t}}+\frac{K_{m 3}\left(1-x_{1}-x_{2}\right)}{K_{m 4}}+x_{2}}+\frac{K_{m 1}}{\frac{K_{m 1}}{S_{t o t}}+\frac{K_{m 1}\left(1-x_{1}-x_{2}\right)}{K_{m 2}}+x_{1}}:=F_{2}\left(x_{1}, x_{2}\right) .
\end{aligned}
$$

It is easy to see that $F_{1}$ is strictly decreasing in $x_{2}$, and $F_{2}$ is strictly decreasing in $x_{1}$ on

$$
K_{0}=\left\{\left(x_{1}, x_{2}\right) \mid x_{1} \geq 0, x_{2} \geq 0, x_{1}+x_{2} \leq 1\right\} .
$$

So, (13) is strongly monotone on some open set $W$ containing $K_{0}$ with respect to the cone

$$
\left\{\left(x_{1}, x_{2}\right) \mid x_{1} \leq 0, x_{2} \geq 0\right\} .
$$

Applying Lemma 2, the flow of (13) has eventually positive derivatives on $K_{0}(\subset W)$, and $\mathbf{A 6}$ is valid.

So the system formulated in the form of (12) satisfies all assumptions A1 to A7. Applying Theorem 1, we have:

Theorem 2 There exist a positive $\varepsilon^{*}<\varepsilon_{0}$ such that for each $\varepsilon \in\left(0, \varepsilon^{*}\right)$, the forward trajectory of (12) starting from almost every point in $D_{\varepsilon}$ converges to some equilibrium.

In fact, since the reduced system is of dimension two, we know that every trajectory in $D_{\varepsilon}$, instead of almost every trajectory in $D_{\varepsilon}$, converges to some equilibrium ([19]).

It is worth pointing out that the conclusion we obtained from the above theorem is only valid for small enough $\varepsilon$; that is, the concentration of the enzyme should be much smaller than the concentration of the substrate. Unfortunately, this is not always true in biological systems, especially when feedbacks are present. However, if the sum of the Michaelis-Menten constants and the total concentration of the substrate are much larger than the concentration of enzyme, a different scaling:

$$
x_{1}=\frac{\left[S_{0}\right]}{A}, \quad x_{2}=\frac{\left[S_{2}\right]}{A}, \quad \varepsilon^{\prime}=\frac{E_{t o t}}{A}, \quad t=\tau \varepsilon^{\prime},
$$

where $A=S_{\text {tot }}+K_{m 1}+K_{m 2}+K_{m 3}+K_{m 4}$ will allow us to obtain the same convergence result.

\subsection{Another example}

The following example demonstrates the importance of the smallness of $\varepsilon$. Consider an $m+1$ dimensional system:

$$
\begin{aligned}
\frac{d x}{d t} & =\gamma\left(y_{1}, \ldots, y_{m}\right)-\beta(x) \\
\varepsilon \frac{d y_{i}}{d t} & =-d_{i} y_{i}-\alpha_{i}(x), \quad d_{i}>0, \quad i=1, \ldots, m .
\end{aligned}
$$

under the following assumptions:

1. There exists an integer $r>1$ such that the derivatives of $\gamma, \beta$, and $\alpha_{i}$ are of class $C_{b}^{r}$ for sufficiently large bounded sets. 
2. The function $\beta(x)$ is odd, and it approaches infinity as $x$ approaches infinity.

3. The function $\alpha_{i}(x)(i=1, \ldots, m)$ is bounded by positive constant $M_{i}$ for all $x \in \mathbb{R}$.

4. The number of roots to the equation

$$
\gamma\left(\alpha_{1}(x), \ldots, \alpha_{m}(x)\right)=\beta(x)
$$

is countable.

We are going to show that on any large enough region, and provided that $\varepsilon$ is sufficiently small, almost every trajectory converges to an equilibrium. To emphasize the need for small $\varepsilon$, we also show that when $\varepsilon>1$, limit cycles may appear.

Assumption 4 implies A7, and because of the form of (14), A3 and A4 follow naturally. A6 also holds, as every one dimensional system is strongly monotone. For A5, we take

$$
D_{\varepsilon}=\left\{(x, y)|| x|\leq a,| y_{i} \mid \leq b_{i}, i=1, \ldots, m\right\},
$$

where $b_{i}$ is an arbitrary positive number greater than $\frac{M_{i}}{d_{i}}$ and $a$ can be any positive number such that

$$
\beta(a)>N_{b}:=\max _{\left|y_{i}\right| \leq b_{i}} \gamma\left(y_{1}, \ldots, y_{m}\right)
$$

Picking such $b_{i}$ and $a$ assures

$$
x \frac{d x}{d t}<0, \quad y_{i} \frac{d y_{i}}{d t}<0
$$

i.e. the vector field points transversely inside on the boundary of $D_{\varepsilon}$. Let $U$ and $V$ be some bounded open sets such that $D_{\varepsilon} \subset U \times V$, and assumption 1 holds on $U$ and $V$. Then A1 and A2 follow naturally. By our main theorem, for sufficiently small $\varepsilon$, the forward trajectory of (14) starting from almost every point in $D_{\varepsilon}$ converges to some equilibrium.

On the other hand, convergence does not hold for large $\varepsilon$. Let

$$
\beta(x)=\frac{x^{3}}{3}-x, \alpha_{1}(x)=2 \tanh x, \quad m=1, \gamma\left(y_{1}\right)=y_{1}, d_{1}=1 .
$$

It is easy to verify that $(0,0)$ is the only equilibrium, and the Jacobian matrix at $(0,0)$ is

$$
\left(\begin{array}{cc}
1 & 1 \\
-2 / \varepsilon & -1 / \varepsilon
\end{array}\right)
$$

When $\varepsilon>1$, the trace of the above matrix is $1-1 / \varepsilon>0$, its determinant is $1 / \varepsilon>0$, so the (only) equilibrium in $D$ is repelling. By the Poincaré-Bendixson Theorem, there exists a limit cycle in $D$.

\section{Conclusions}

Singular perturbation techniques are routinely used in the analysis of biological systems. The geometric approach is a powerful tool for global analysis, since it permits one to study the behavior for finite $\varepsilon$ on a manifold in which the dynamics is "close" to the slow dynamics. Moreover, and most relevant to us, a suitable fibration structure allows the "tracking" of trajectories and hence the lifting to the full system of the exceptional set of non-convergent trajectories, if the slow system satisfies the conditions of Hirsch's Theorem. Using the geometric approach, we were able to provide a global convergence theorem for singularly perturbed strongly monotone systems, in a form that makes it applicable to the study of double futile cycles. 


\section{Acknowledgment}

We have benefited greatly from correspondence with Christopher Jones and Kaspar Nipp about geometric singular perturbation theory. We also wish to thank Alexander van Oudenaarden for questions that triggered much of this research, and David Angeli, Thomas Gedeon, and Hal Smith for helpful discussions.

This work was supported in part by NSF Grant DMS-0614371.

\section{References}

[1] Abed, E.H., "Singularly perturbed Hopf bifurcation," IEEE Trans. Circuits \& Systems 32 (1985), pp. $1270-1280$.

[2] Asthagiri, A.R., Lauffenburger, D.A., "A computational study of feedback effects on signal dynamics in a mitogen-activated protein kinase (MAPK) pathway model," Biotechnol. Prog. 17 (2001), pp. $227-239$.

[3] Bijlsma, J.J., Groisman, E.A., "Making informed decisions: regulatory interactions between twocomponent systems," Trends Microbiol 11 (2003), pp. 359-366.

[4] Burack, W.R., Sturgill, T.W., "The Activating dual phosphorylation of MAPK by MEK is nonprocessive," Biochemistry 36 (1997), pp. 5929-5933.

[5] Chang, L., Karin, M., "Mammalian MAP kinase signaling cascades," Nature 410 (2001), pp. 37-40.

[6] Chen, H., Bernstein, B.W., Bamburg, J.R., "Regulating actin filament dynamics in vivo," Trends Biochem. Sci. 25 (2000), pp. 19-23.

[7] Chisci, L., Falugi, P., "Asymptotic tracking for state-constrained monotone systems," Proc. 44th IEEE Conf. Decision and Control Seville, 2005, paper ThC17.5.

[8] Christofides, P.D., Teel, A.R., "Singular perturbations and input-to-state stability," IEEE Trans. Automat. Contr 41 (1996), pp. 1645-1650.

[9] Dancer, E.N., "Some remarks on a boundedness assumption for monotone dynamical systems," Proc. of the AMS 126(1998), pp. 801-807.

[10] Donovan, S., Shannon, K.M., Bollag, G., "GTPase activating proteins: critical regulators of intracellular signaling," Biochim. Biophys Acta 1602 (2002), pp. 23-45.

[11] Edelstein-Keshet, L., Mathematical Models in Biology, McGraw-Hill, 1988

[12] Fenichel, N., "Geometric singular perturbation theory for ordinary differential equations," J. of Differential Equations 31(1979), pp. 53-98.

[13] Ferrell, J.E., Bhatt, R.R., "Mechanistic studies of the dual phosphorylation of mitogen-activated protein kinase," J. Biol. Chem. 272(1997), pp. 19008-19016.

[14] Gardner, T.S., Cantor, C.R., Collins, J.J., "Construction of a genetic toggle switch in Escherichia coli.," Nature 403 (2000), pp. 339-342

[15] Grossman, A.D., "Genetic networks controlling the initiation of sporulation and the development of genetic competence in Bacillus subtilis," Annu Rev Genet. 29 (1995), pp. 477-508. 
[16] Gunawardena, J., "Multisite protein phosphorylation makes a good threshold but can be a poor switch," Proc. Natl. Acad. Sci. 102 (2005), pp. 14617-14622.

[17] Hirsch, M., "Systems of differential equations that are competitive or cooperative II: Convergence almost everywhere," SIAM J. Mathematical Analysis 16(1985), pp. 423-439.

[18] Hirsch, M., "Differential equations and convergence almost everywhere in strongly monotone flows," Contemporary Mathematics 17(1983), pp. 267-285.

[19] Hirsch, M., Smith, H.L., "Monotone dynamical systems," in Handbook of Differential Equations, Ordinary Differential Equations: Volume 2, Elsevier, Amsterdam, 2005.

[20] Huang, C.-Y.F., Ferrell, J.E., "Ultrasensitivity in the mitogen-activated protein kinase cascade," Proc. Natl. Acad. Sci. USA 93 (1996), pp. 10078-10083.

[21] Isidori, A., Nonlinear Control Systems: An Introduction, Springer-Verlag, London, Third Edition, 1995.

[22] Jiang, J.F., "On the global stability of cooperative systems," Bulletin of the London Math Soc 6(1994), pp. $455-458$.

[23] Jones, C.K.R.T., "Geometric singular perturbation theory," in Dynamical Systems (Montecatini. Terme, 1994), Lect. Notes in Math. 1609, Springer-Verlag, Berlin.

[24] Karp, G., Cell and Molecular Biology, Wiley, New York 2002.

[25] Knobloch, K.W., Aulbach, B., "Singular perturbations and integral manifolds," J. Math. Phys. Sci. 13 (1984), pp. 415-424.

[26] Lew, D.J., Burke, D.J., "The spindle assembly and spindle position checkpoints," Annu Rev Genet. 37 (2003), pp. 251-282.

[27] Markevich, N.I., Hoek, J.B., Kholodenko, B.N., "Signaling switches and bistability arising from multisite phosphorylation in protein kinase cascades," J. Cell Biol. (2004), 164 pp. 353-359.

[28] Moreau, L., Stability of continuous-time distributed consensus algorithms, Proc. 43rd IEEE Conf. Decision and Control, Paradise Island, Bahamas, 2004, paper ThC10.4.

[29] Murray, J.D., Mathematical Biology, 3rd Edition, Springer, New York, 2002.

[30] Nipp, K., "Smooth attractive invariant manifolds of singularly perturbed ODE's," Research Report, No. 92-13 (1992),

[31] Ortega, F., Garcés, J., Mas, F., Kholodenko, B.N., Cascante, M., "Bistability from double phosphorylation in signal transduction: Kinetic and structural requirements," FEBS J 273 (2006), pp. 3915-3926.

[32] Pomerening, J.R., Sontag, E.D., Ferrell Jr., J.E., "Building a cell cycle oscillator: hysteresis and bistability in the activation of Cdc2," Nature Cell Biology 5(2003), pp. 346-351.

[33] Sakamoto, K., "Invariant manifolds in singular perturbation problems for ordinary differential equations," Proceedings of the Royal Society of Edinburgh 116A (1990), pp. 45-78. 
[34] Samoilov, M., Plyasunov, S., Arkin, A.P., "Stochastic amplification and signaling in enzymatic futile cycles through noise-induced bistability with oscillations," Proc Natl Acad Sci USA 102 (2005), pp. 2310-2315.

[35] Sel'kov, E.E., "Stabilization of energy charge, generation of oscillation and multiple steady states in energy metabolism as a result of purely stoichiometric regulation," Eur. J. Biochem 59(1) (1975), pp. 151-157.

[36] Sha, W., Moore, J., Chen, K., Lassaletta, A.D., Yi, C.S., Tyson, J.J., Sible, J.C., "Hysteresis drives cell-cycle transitions in Xenopus laevis egg extracts," Proc. Natl. Acad. Sci. 100 (2003), pp. 975-980.

[37] Smith, H.L., Monotone dynamical systems: An introduction to the theory of competitive and cooperative systems, Mathematical Surveys and Monographs, vol. 41, (AMS, Providence, RI, 1995).

[38] Sontag, E.D., Mathematical Control Theory: Deterministic Finite Dimensional Systems, Springer, New York, 1990. Second Edition, 1998.

[39] Sontag, E.D., "Some new directions in control theory inspired by systems biology," Systems Biology $\mathbf{1}(2004)$, pp. 9-18.

[40] Sontag, E.D., "Molecular systems biology and control," European J. Control 11 (2005), pp. 396-435.

[41] Stryer, L., Biochemistry, Freeman, New York 1995.

[42] Sulis, M.L., Parsons, R., "PTEN: from pathology to biology," Trends Cell Biol. 13 (2003), pp. 478-483.

[43] Teel, A.R., Moreau, L., Nesic, D., "A unification of time-scale methods for systems with disturbances," IEEE Trans. Automat. Contr 48 (2003), pp. 1526-1544.

[44] Wang, L., Sontag, E.D., "A Remark on Singular Perturbations of Strongly Monotone Systems," Proc. IEEE Conf. Decision and Control San Diego, Dec. 2006, pages WeB10.5.

[45] Wang, L., Sontag, E.D., "On the number of steady states in phosphorylation/dephosphorylation cycles," in preparation.

[46] Widmann, C., Spencer, G., Jarpe, M.B., Johnson, G.L., "Mitogen-activated protein kinase: Conservation of a three-kinase module from yeast to human," Physiol. Rev. 79 (1999),, pp. 143-180.

[47] Zhao, Y., Zhang, Z.Y., "The mechanism of dephosphorylation of extracellular signal-regulated kinase 2 by mitogen-activated protein kinase phosphatase 3," J. Biol. Chem. 276 (2001), pp. 32382-32391. 